\title{
$\epsilon$ Aur in eclipse: Post-AGB primary and disk-shaped secondary
}

\section{Chinnathambi Muthumariappan ${ }^{1}$, Mudumba Parthasarathy ${ }^{2,3,4}$, Kanniah Jayakumar ${ }^{1}$ and Manickam Appakutty ${ }^{1}$}

\author{
${ }^{1}$ Vainu Bappu Observatory, Indian Institute of Astrophysics, Kavalur, 635701 India \\ email: muthu@iiap.res.in \\ ${ }^{2}$ National Astronomical Observatory of Japan 2-21-1 Osawa, Mitaka, Tokyo 181-8588, Japan \\ ${ }^{3}$ McDonnell Center for the Space Sciences, Department of Physics Washington University in \\ St. Louis, One Brookings Drive, St. Louis, MO 63130, USA \\ ${ }^{4}$ Aryabhatta Research Institute of Observational Sciences Nainital - 263 129, India
}

\begin{abstract}
We present physical and chemcal properties of the disk in the eclipsing binary system $\epsilon$ Aur by solving $2 \mathrm{D}$ radiative transfer problem. We also present preliminary results of our high resolution spectroscopic monitoring of $\mathrm{K} \mathrm{I}, \mathrm{Na}$, and $\mathrm{H} \alpha$ line profiles variation during the totality phase of the recent eclipse of $\epsilon$ Aur.
\end{abstract}

Keywords. (stars:) binaries: eclipsing, (stars:) circumstellar matter, radiative transfer

\section{Introduction}

$\epsilon$ Aur is a eclipsing binary with a period of 27.1 years which is punctuated by flat bottomed eclipse of two years duration. IR observations showed an excess emission consistent with the presence of a dusty disk. The optical spectra of $\epsilon$ Aur near the end of 1954-1956 eclipse was used to hypothesis the presence of a Be like star at the center of a large disk (Hack 1961). From the SED constructed with their new Spitzer Space telescope observations and with the archival far-UV to mid-IR data, Hoard et al. (2010) proposed a three-component model for the $\epsilon$ Aur system which consist of a F-type post-AGB star of mass $2.2 \mathrm{M}_{\odot}$ and a B5V type main sequence star surrounded by a geometrically thin and partially transparent disk of size 3.8 AU which is viewd edge-on. Their model deals with average bulk properties of the disk with a single-temperature black body of $550 \mathrm{~K}$ and an uniform mass distribution. Using the results obtained by Kloppenborg et al. (2010) by their interferometric imaging, we attempt to make a detailed disk model by fitting the SED by solving radiative transfer problem in two dimensional case.

\section{SED and $2 \mathrm{D}$ radiative transfer models}

We have used the archival data viz, the UV data from HST GHRS, optical and nearIR photometric data from SIMBAD, and mid-IR and far-IR photometric measurements were from IRAS and MSX space missions for constructing the SED. We used 2D MonteCarlo radiative transfer code SRCDUST, based on the techniques developed by Bjorkman \& Wood (2001). We considered a F0Ia primary, B5V secondary and a flared disk with different dust composition as the input and the disk temperature structure and model SED were obtained as the output. Our results show that grains in the disk are much larger $\left(a_{\mathrm{min}}=10 \mu \mathrm{m}, a_{\max }=100 \mu \mathrm{m}\right)$ than the ISM grains. In addition, larger carbon grain composition appears to fit the SED better than the larger amorphous silicate grain composition (Fig. 1). This may show that the disk was resulted from a mass transfer 

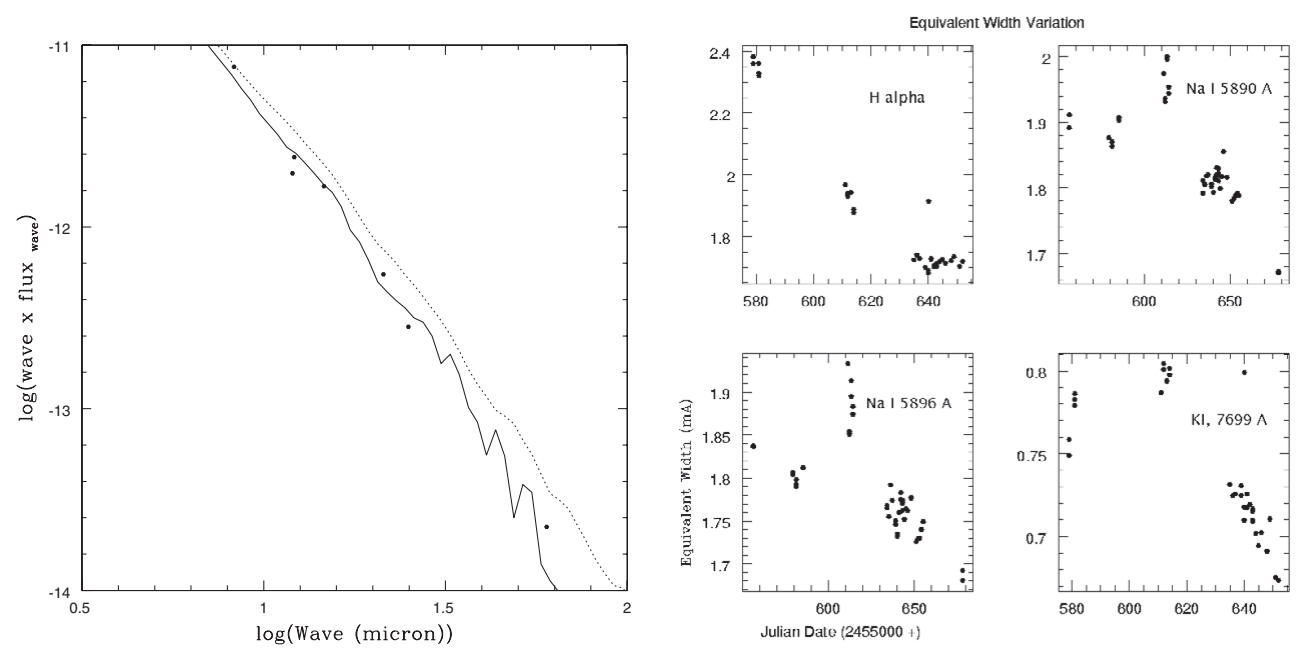

Figure 1. Left: radiative transfer model SEDs of the disk with a) amorphous carbon alone (solid line) b) amorphous silicate alone (dotted line). Right: equivalent width variation of spectral lines.

from post-AGB primary. The disk is physically thin and is seen nearly edge on, and the disk mass is smaller than $\sim 0.0005 \mathrm{M}_{\odot}$.

\section{Optical Spectroscopic Monitoring}

Spectroscopic monitoring of $\epsilon$ Aur was carried out at the Vainu Bappu Telescope, Kavalur, India from 25th December 2010 to 26th April 2011. About 40 spectra were taken with a resolution of either 70000 or 30000 covering the full optical domain. Spectra were reduced and calibrated with IRAF. Line profiles showed significant variations both in their shapes and in radial velocities. $\mathrm{H} \alpha$ shows a red wing emission however the blue wing emission was not detected. Equivalent widths of these four spectral lines were obtained from all spectra and are plotted against the Julian date in Fig. $1 . \mathrm{H} \alpha$ variation is quite different from other three lines. The absorption line strength variation in K I line during the eclipse clearly shows that the disk shaped secondary is having an extended neutral gaseous envelope which was first discovered by Parthasarathy \& Lambert (1983). The shell component of absorption lines (Fig. 1) have decreased in strength by the time of 4th contact. A sudden jump in the EW of $\mathrm{H} \alpha$ and KI lines were observed at JD 2155640 indicating enhanced gas density, or dense gas ring or clumpiness in the disk.

\section{Acknowledgements}

MP is thankful to Prof. Shoken Miyama, Prof. Ramanath Cowsik, and Prof. Yoichi Takeda for their kind encouragement and support.

\section{References}

Bjorkman, J. E. \& Wood, K. 2001, ApJ, 554, 615

Hack, M. 1961, MemSAI 32, 351

Hoard, D. W. \& Howell, S. B., Stencel R. E. 2010, ApJ, 714, 549

Kloppenbourg, B., Robert, S., \& Monnier, J. D., et al. 2010, Nature, 464, 870

Parthasarathy, M. \& Lambert, D. L. 1983, IAU Circular 3766 